EPJ Web of Conferences 71, 00031 (2014)

DOI: 10.1051/epjconf/20147100031

(C) Owned by the authors, published by EDP Sciences, 2014

\title{
Impact of LHC Run-1 on particle astrophysics
}

\author{
David d'Enterria ${ }^{1, a}$ \\ ${ }^{1}$ CERN, PH Department, $\mathrm{CH}-1211$ Geneva 23, Switzerland
}

\begin{abstract}
A review of the impact of the first three years of LHC operation on two of the most important open questions in astroparticle physics is presented. Measurements in proton-proton collisions at the energy frontier that provide valuable information on the identity of the highest-energy particles in the cosmos as well as new constraints on the nature of dark matter, are summarized.
\end{abstract}

\section{Introduction}

The nature of the dark matter that pervades the universe [1] as well as the identity and origin of the highest-energy cosmic rays reaching Earth [2] are among the most pressing open questions in particle astrophysics today. During the last 3 years, the CERN Large Hadron Collider (LHC) has been colliding protons (and nuclei) at the highest center-of-mass energies ever studied in the laboratory (up to $\sqrt{s}=8 \mathrm{TeV}$ ). Many measurements carried out by the different experiments provide new insights on the solution of both open issues. On the one hand, detailed LHC studies of the theory of the strong interaction (quantum chromodynamics, QCD) have resulted in valuable constraints on the Monte Carlo models used to interpret the extended air-showers produced by ultra-high-energy cosmic rays (UHECR) colliding with air nuclei in the upper atmosphere. On the other, searches of dark matter (DM) particles -which escape the LHC detectors but can be discovered as an excess of events with missing transverse energy- have been carried out in different final-states corresponding to various visible particles accompanying DM production. The latest developments in UHECR and DM physics as well as the most relevant LHC measurements providing information on both key problems are summarized hereafter.

\section{Impact of LHC data on ultra-high-energy cosmic rays}

The highest energy hadronic interactions on Earth occur in collisions of cosmic rays -protons and nuclei produced in various astrophysical sources- with air nuclei as they enter the atmosphere [2]. The highest cosmic-ray energies measured are of the order of $10^{20} \mathrm{eV}$ (Fig. 1, left), corresponding to the Greisen-Zatsepin-Kuzmin (GZK) cutoff of CRs propagating through intergalactic space and dissociating in collisions with the microwave background [3] and/or to the maximum energy reachable in astrophysical accelerators [4]. The experimental determination of the primary UHECR energy and mass relies upon the comparison of the properties of the produced extensive air-showers

a e-mail: dde@cern.ch

This is an Open Access article distributed under the terms of the Creative Commons Attribution License 2.0, which permits unrestricted use, distribution, and reproduction in any medium, provided the original work is properly cited. 

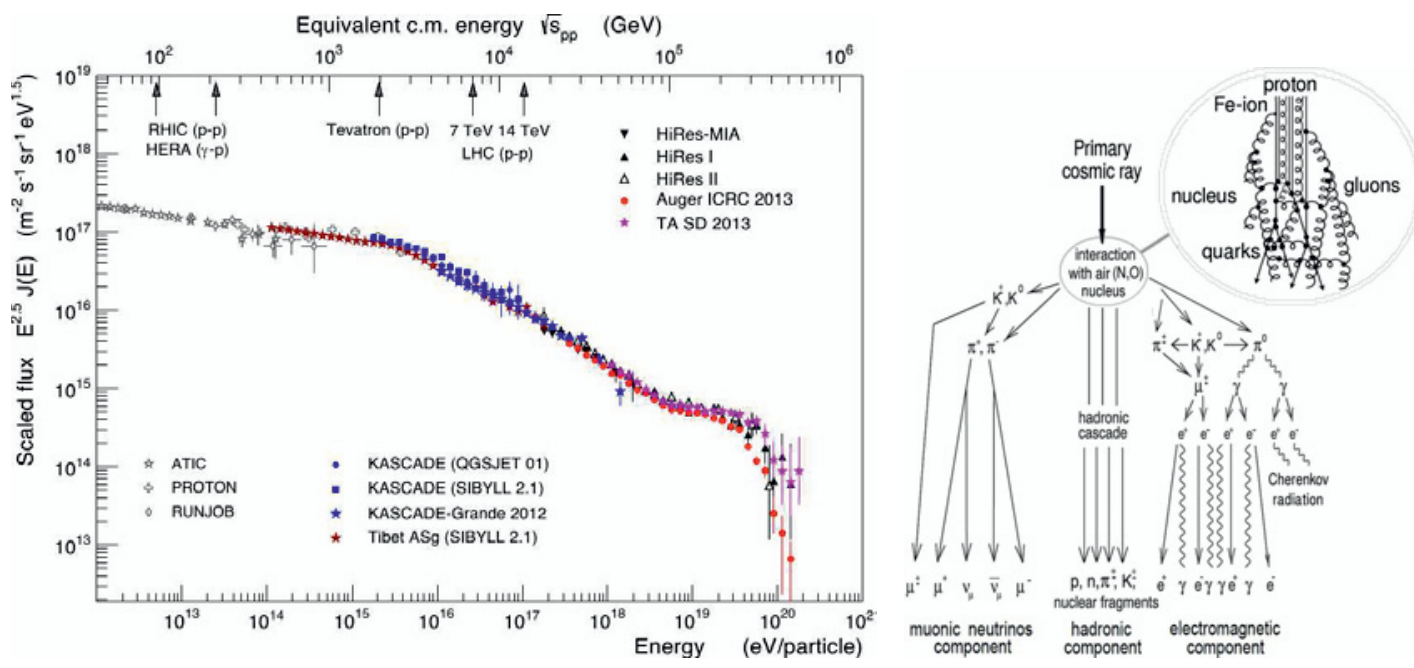

Figure 1. Left: Cosmic rays flux (scaled by $\mathrm{E}_{\mathrm{CR}}{ }^{2.5}$ ) as a function of $\mathrm{CR}$ energy (equivalent c.m. energies for various colliders are shown on the top axis) [11]. Right: Schematic "microscopic" view of an extensive air shower produced by an ultrarelativistic cosmic ray (proton or Fe-ion) colliding with a nucleus in the upper atmosphere.

(EAS, Fig. 1 right) -such as the shower peak position $\mathrm{X}_{\max }$, and the number of electrons and muons on ground $\mathrm{N}_{\mathrm{e}, \mu}$ - to Monte Carlo (MC) hadronic simulations: EPos [5, 6], QGSJET01 [7], QGSJET-II [8] and SIBYLL [9]. The dominant source of uncertainty in the interpretation of the EAS data stems from our limitations to model particle production in strongly-interacting systems at c.m. energies up to $\sqrt{s_{\mathrm{GZK}}} \approx 400 \mathrm{TeV}$, i.e. more than two orders of magnitude higher than those studied at particle colliders before the LHC. Indeed, even at asymptotically high energies the collision between two hadronic objects is sensitive to non-perturbative (hadronization, beam remnants, soft peripheral diffractive scatterings) as well as semihard (saturation of gluon densities, multiparton interactions) dynamics that need to be directly constrained from experimental data [10].

The LHC has extended by more than a factor of three the c.m. energies for which we have direct $\mathrm{p}$-p measurements available, going beyond the "knee" structure of the $\mathrm{CR}$ spectrum at $\mathrm{E}_{\mathrm{CR}} \approx 10^{15.5} \mathrm{eV}$ (Fig. 1, left). The following LHC inclusive observables are sensitive to the non-perturbative and semihard QCD dynamics implemented in hadronic MCs commonly used in UHECR physics:

- Inelastic p-p cross section $\sigma_{\text {inel }}$. Hadronic cross sections are not directly computable from the QCD Lagrangian ${ }^{1}$, but are constrained by basic quantum mechanical relations (such as the Froisart bound, the optical theorem, and dispersion relations) which can be combined with experimental data to make predictions. The measured inelastic p-p cross section, $\sigma_{\text {inel(visible })}=73(60) \pm 2 \mathrm{mb}$ at $\sqrt{s}=7 \mathrm{TeV}$ [13-16], was mostly overpredicted by the MCs (Fig. 2, left), which tended to over (under) estimate the diffractive contributions at high (low) masses. The measured value of $\sigma_{\text {inel }}$ at the LHC implies a reduced $\sigma_{\text {inel }}\left(\mathrm{p}\right.$-Air) cross section and subsequently a deeper $\mathrm{X}_{\max }$ shower position of UHECR.

- Pseudorapidity density of charged particles at midrapidity $\mathrm{dN}_{\mathrm{ch}} /\left.\mathrm{d} \eta\right|_{\eta=0}$ and event-by-event distribution of the charged particle multiplicity $\mathrm{P}\left(\mathrm{N}_{\mathrm{ch}}\right)$. At LHC energies about $70 \%$ of the produced

\footnotetext{
${ }^{1}$ Although the possibility of using lattice QCD calculations [12] one day, should not be discarded.
} 

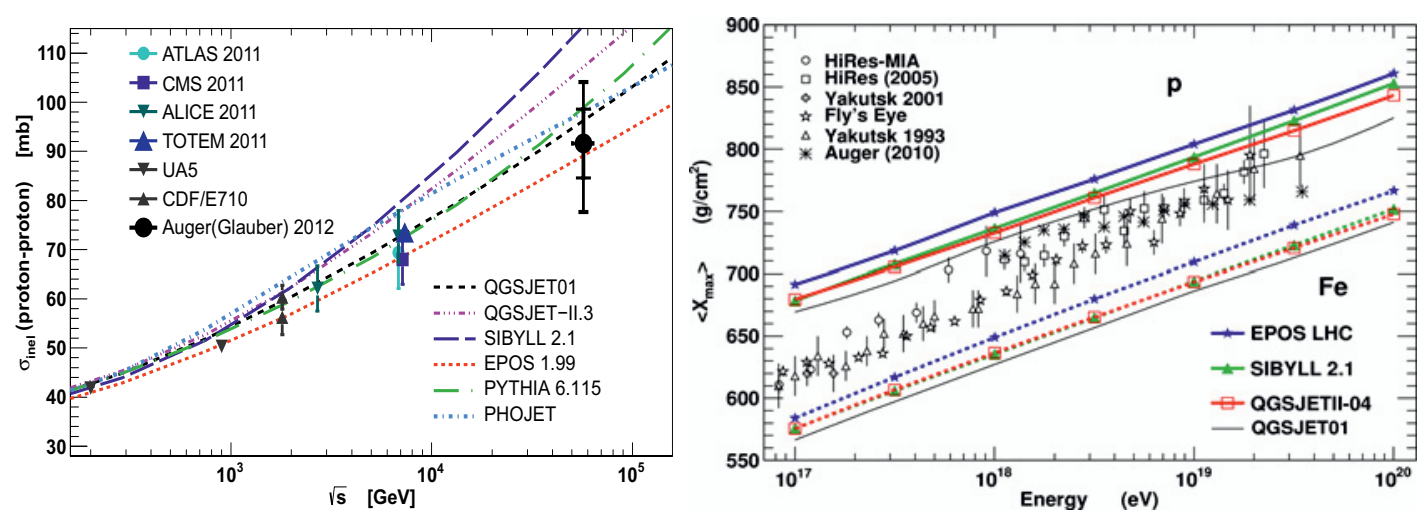

Figure 2. Left: Inelastic p-p cross sections measured at colliders and estimated by the P. Auger Observatory at $\sqrt{s} \approx 50 \mathrm{TeV}$ [21], compared to hadronic MC predictions. Right: Measured mean $\mathrm{X}_{\max }$ position as a function of $\mathrm{CR}$ energy, compared to hadronic MC predictions for proton- and Fe-induced showers in the atmosphere [11].

hadrons come from (multi)parton interactions (with exchanged momenta of order 1-2 GeV) followed by their fragmentation into hadrons. The measurements of $\mathrm{dN}_{\mathrm{ch}} /\left.\mathrm{d} \eta\right|_{\eta=0}$ and $\mathrm{P}\left(\mathrm{N}_{\mathrm{ch}}\right)$ have been crucial to improve the modeling of multiparton interactions (at large values of $\mathrm{N}_{\mathrm{ch}}$ ) and diffraction (for low $\mathrm{N}_{\mathrm{ch}}$ ). None of the pre-LHC CR hadronic models predicted precisely both distributions, although taken together they "bracketed" the experimental data [10].

- Energy distribution of (very) forward particles. Knowledge of the particle and energy flows emitted at very small polar angles in hadronic collisions - dominated by underlying event activity, beam remnants and diffractive fragments- is also crucial for understanding the first stages of the EAS development. Most of the UHECR models reproduced appropriately the data at pseudorapidities $|\eta|=3-5$ [17-19], but less so for the neutral activity close to the beam rapidity [20].

Two main conclusions can be extracted from the confrontation of data to pre-LHC MCs [10]. First, although no model reproduced consistently all results, when taken together they "bracketed" the LHC data and could be globally trusted to interpret the EAS properties. Second, the absence of a drastic change in the properties of multiparticle production at the LHC (corresponding to energies $\mathrm{E}_{\mathrm{CR}} \approx 10^{16} \mathrm{eV}$ ), confirmed that the CR "knee" at $10^{15.5} \mathrm{eV}$ is not due to the production of new (unobserved) particles, as speculated in some cases, but to the change from a light to a more heavy mass $\mathrm{CR}$ composition. The update of the event generators to account for the LHC measurements has led to a convergence of their predictions: the $\mathrm{X}_{\max }$ slope has decreased (increased) for EPOS (QGSJET-II) and their $\mathrm{N}_{\mu}$ values have raised at the highest $\mathrm{CR}$ energies (although they are still below the measured $\mathrm{N}_{\mu}$ ). Around the GZK cutoff, the CR data prefer a mixed proton-iron composition (Fig. 2, right), with reduced model uncertainties (from $\sim 50 \mathrm{~g} / \mathrm{cm}^{2}$ to $\sim 20 \mathrm{~g} / \mathrm{cm}^{2}$ for values of $X_{\max }$ where the proton-iron difference is about $\left.100 \mathrm{~g} / \mathrm{cm}^{2}\right)$ [11].

\section{Impact of LHC data on dark matter searches}

Dark matter is required to explain many astrophysical and cosmological observations inferred from its gravitational effects on visible matter, radiation, and the large-scale structure of the universe. The astronomical evidences for DM have grown steadily since (i) the observed faster-than-Kleperian rotation curves of stars in the Milky Way (in the 1930s) and, in general, in spiral galaxies (in the 

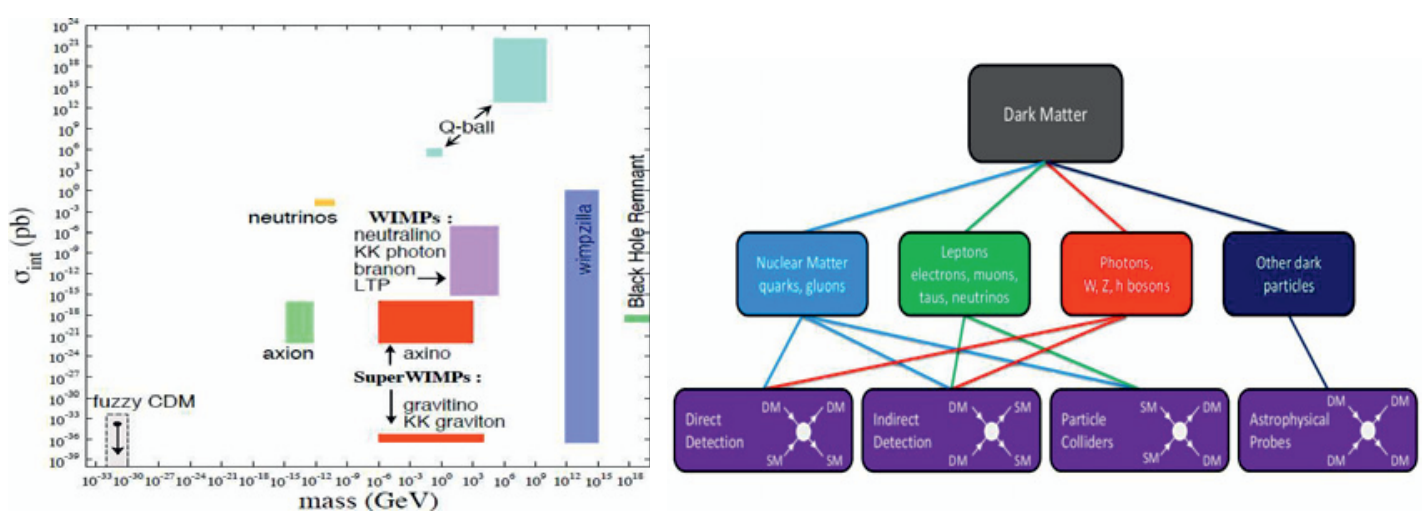

Figure 3. Left: DM particle candidates in the DM-nucleon interaction cross section vs. DM-mass plane [27]. Right: Dark matter detection approaches (depending on its interaction with SM and other DM particles): direct in underground experiments, indirect in space detectors, particle colliders, and astrophysical probes [1].

late 1970s); strongly supported by (ii) the collision of galaxies in the Bullet Cluster [22], where gravitational-lensing reveals interaction-less galaxies lying ahead of the visible collisional gas; and more recently among others by e.g. (iii) the relative motion between the Milky Way and Andromeda, which indicates a combined virial mass $\mathrm{M}_{\text {virial }}=3.2 \cdot 10^{12} \times \mathrm{M}_{\odot}$, ten times larger than that estimated from the visible stars and gas [23]. The existence of DM is also supported by many cosmological observations such as (i) the large-scale structure of the universe given by galaxy distribution surveys confronted to statistical simulations [24]; (ii) the peak heights of the cosmic microwave background measured by WMAP and Planck $[25,26]$ (lower than that expected in the absence of DM); plus (iii) other measures of matter density (baryon acoustic oscillations, big bang nucleosyntesis, supernova distances, etc.). The post-Planck global fit of the energy budget of the universe yields a $26.8 \% \mathrm{DM}$ content, yielding a local halo density $\rho_{\mathrm{DM}} \approx 0.3 \mathrm{GeV} / \mathrm{cm}^{3}$ with average speed $\bar{v}_{\mathrm{DM}}$ of a few hundred $\mathrm{km} / \mathrm{s}$, and a flux of $10^{5} \mathrm{~cm}^{-2} \mathrm{~s}^{-1}$ on Earth.

DM particles are gravitationally-interacting, stable, massive, cold, non baryonic, and an early universe thermal relic. Weakly interacting massive particles (WIMP) beyond the Standard Model (SM), constitute paradigmatic candidates with such properties. For masses $\mathrm{m}_{\chi}=10 \mathrm{GeV}-1 \mathrm{TeV}$ and typical weak interaction $\chi$-SM cross sections in the pb-range $\left(\sigma_{\chi-\mathrm{SM}} \approx \sigma_{\mathrm{EW}}\right)$, one obtains the observed density $\Omega_{\mathrm{DM}} \propto \mathrm{m}_{\chi}^{2} / g_{\mathrm{EW}}^{4} \sim \mathrm{O}(10 \%)$, a fact often referred to as the "WIMP miracle". Alternative viable possibilities exist, however, with lower and/or higher $\mathrm{m}_{\chi}$ and $\sigma_{\chi \text {-sм }}$ values (Fig. 3, left) [27]. Popular theoretical extensions of the SM provide DM candidates such as (R-parity conserving) SUSY with a stable lightest supersymmetric particle (LSP, such as the neutralino or the gravitino), or the lightest Kaluza-Klein tower in extra-dimensions models. Other possibilities include heavy R-handed or sterile neutrinos, axions, or new particles of an unknown hidden sector. Apart from the astrophysical probes, three complementary DM search strategies exist with different generic combinations of DM and SM particles in the final- and initial-states (Fig. 3, right):

- Direct detection in underground experiments sensitive to $\chi$-nucleon scattering $(\chi \mathrm{N} \rightarrow \chi \mathrm{N})$ : The signals sought are anomalous nuclear recoils of order $\mathrm{E}_{\mathrm{R}}=N \cdot \rho_{\mathrm{DM}} \cdot \sigma_{\mathrm{DM}-\mathrm{SM}} \cdot \bar{v}_{\mathrm{DM}} \approx 10 \mathrm{keV}$. Ultralow-background scintillation/ionization/phonon detectors are required with sensitivities above 1 event $/ 100 \mathrm{~kg} /$ year. The current limits in the WIMP-nucleon (spin-independent) interaction are 

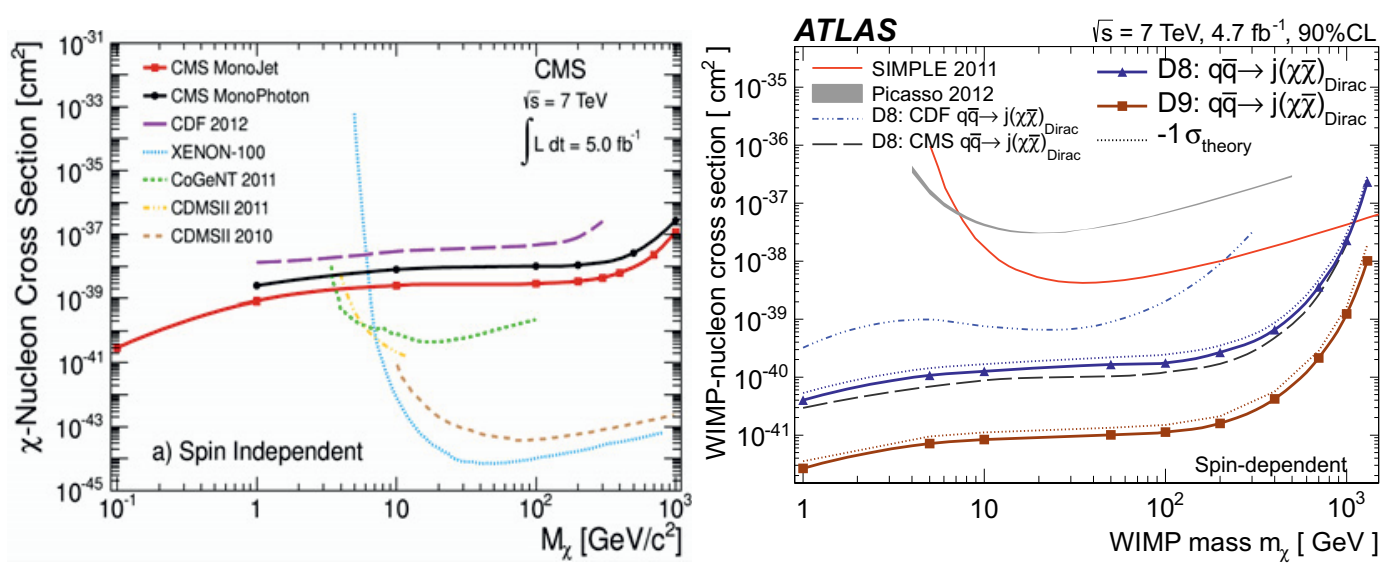

Figure 4. Exclusion curves in $\chi$-N interaction cross-section vs. $\chi$-mass for spin-independent (left) and spindependent (right) models for various underground, space, and LHC (monojet and monophoton) searches [35, 36].

approaching $\sigma_{\chi-\mathrm{N}} \approx 10^{-45} \mathrm{~cm}^{2}$ (i.e. the $\mathrm{zb}$ range!) for masses $\mathrm{m}_{\chi} \approx 50 \mathrm{GeV}$ (Fig. 4, left), although there have been conflicting (non-reproducible) signals consistent with $\sigma_{\chi-\mathrm{N}} \approx 10^{-41} \mathrm{~cm}^{2}$ at lower masses $\mathrm{m}_{\chi} \approx 10-50 \mathrm{GeV}$ by different experiments (DAMA, CRESST) as well as not yet statistically-significant excesses in the same region (CDMS).

- Indirect detection in space detectors sensitive to SM-pairs from DM-DM annihilation $(\chi \bar{\chi} \rightarrow$ $\mathrm{X}_{\mathrm{SM}} \overline{\mathrm{X}}_{\mathrm{SM}}$ and/or DM decays $\left(\chi \rightarrow \mathrm{X}_{\mathrm{SM}} \overline{\mathrm{X}}_{\mathrm{SM}}\right)$. The typical annihilation cross sections for thermal relic WIMPs are of the order of $\sigma \cdot \bar{v}_{\chi} \approx 3 \cdot 10^{-26} \mathrm{~cm}^{3} / \mathrm{s}$ and final-states with photons (FERMI, Veritas), neutrinos (IceCube), or cosmic rays (Pamela, AMS) are being searched. Some excesses with respect to the SM expectations have been seen e.g. in the PAMELA and AMS cosmic-ray positron fluxes above a few tens of $\mathrm{GeV}[28,29]$, as well as in the $130-\mathrm{GeV}$ line of the FERMI $\gamma$-ray fluxes from the galaxy center [30]. DM interpretations are, however, complicated by the existing uncertainties in the astrophysical propagation of the detected particles and/or by possible extra background sources (pulsars, unaccounted SM contributions, etc.).

- Collider searches through DM (pair) production in the final state (e.g. $\mathrm{p} \mathrm{p} \rightarrow \chi \bar{\chi}+\mathrm{X}_{\mathrm{sM}}$ ). The typical DM collider signature is large missing transverse energy (MET) from undetected LSP coming from the decay cascade of heavier SUSY particles, (often) accompanied with multiple jets and/or leptons. Almost all SUSY searches at the LHC rely on extra MET from a stable $\chi$, for which no evidence has been found so far [31, 32]. Similarly, the first negative results of invisible Higgs decays to DM-pairs with masses $\mathrm{m}_{\chi}<\mathrm{m}_{\mathrm{H}} / 2$-looking at excesses in the MET distribution of associated Higgs $+\mathrm{Z}$-boson production- provide limits in the branching ratio: $\mathrm{BR}(\mathrm{HZ} \rightarrow \chi \bar{\chi} l l)<65 \%$ [33] and 75\% [34]. The most generic searches of DM-pair production in final states with large MET plus initial-state QCD or QED radiation leading to unbalanced mono-jet, mono-photon (or mono-lepton) topologies, are discussed next in more detail.

Generic DM searches at the LHC have looked for mono-jet [35, 36] and mono-photon [37, 38] excesses above the corresponding SM backgrounds. The dominant SM processes producing such topologies are $Z(v v)+j, \gamma(\sim 70 \%)$ and $W\left(v l_{\text {escape }}\right)+j, \gamma(\sim 30 \%)$, since other electroweak and QCD backgrounds can be efficiently removed applying vetoes in isolated leptons and $\Delta \phi$ azimuthal balance cuts. The resulting mono- $j, \gamma$ excesses (or lack thereof) can then be interpreted within generic effective field 
theories for a contact (i.e. mediated by a heavy particle $M_{*}$ ) SM-DM interaction characterized by 2 parameters: $\mathrm{m}_{\chi}$ (the mass of the DM particle, usually considered a Dirac fermion) and $\Lambda=\mathrm{M}_{*} / \sqrt{g_{\chi} g_{\mathrm{q}, \mathrm{g}}}$ (the scale of the effective interaction, with $g_{\chi}$ and $g_{\mathrm{q}, \mathrm{g}}$ the couplings of the mediator to the WIMP and to quark/gluons). The corresponding interaction cross sections, $\sigma(\chi \mathrm{N} \rightarrow \chi \mathrm{N}) \propto\left(\mathrm{g}_{\chi}^{2} \mathrm{~g}_{\mathrm{q}, \mathrm{g}}^{2} / \mathrm{M}_{*}^{4}\right) \cdot \mu_{\chi \mathrm{N}}^{2}$ where $\mu_{\chi \mathrm{N}}$ is the reduced mass of DM-nucleon system, are derived for two types of DM-SM couplings: spin-independent (SI) and spin-dependent (SD). The current LHC exclusion limits (90\% CL) in the $\sigma_{\chi-\mathrm{N}}$ versus $\mathrm{m}_{\chi}$ are shown in Fig. 4 for SI (left) and SD (right) couplings in monojet and monophoton searches [35, 36]. Dark matter-nucleon scattering cross section are excluded above $\sigma_{\chi-\mathrm{N}} \approx 10^{-39} \mathrm{~cm}^{2}$ (SI) and $\sigma_{\chi-\mathrm{N}} \approx 10^{-41} \mathrm{~cm}^{2}$ (SD) for masses $\mathrm{m}_{\chi} \approx 0.1-10 \mathrm{GeV}$. Within the approximations of the underlying effective field theory, these are the most constraining limits for a dark matter particle with mass below $\mathrm{m}_{\chi}=3.5 \mathrm{GeV}$ for spin-independent interactions, a region unexplored by direct detection experiments. For the spin-dependent model, these are the most stringent constraints over the $\mathrm{m}_{\chi}=0.1-200 \mathrm{GeV}$ mass range.

\section{Summary}

The latest developments in ultra-high-energy cosmic-rays (UHECR) and dark matter (DM) physics have been succinctly summarized. Results from the first three years of LHC operation with protonproton collisions up to $8 \mathrm{TeV}$ that provide new insights and constraints on the nature of both particle astrophysics phenomena, have been reviewed.

In the UHECR domain, numerous LHC studies of the non-perturbative and semihard QCD regime have confirmed, first, that the "knee" at $\mathrm{E}_{\mathrm{CR}} \sim 10^{15.5} \mathrm{eV}$ is not due to the production of new (unobserved) particles but to a change from light to a more heavy CR composition [10]. Second, the retuning of the cosmic-rays hadronic MCs using LHC data supports a mixed composition of proton and heavier ions ( $\alpha$ particles and/or iron) at the tail of the cosmic ray spectrum up to $\mathrm{E}_{\mathrm{CR}} \sim 10^{20} \mathrm{eV}$, with reduced model uncertainties (from $\sim 50 \mathrm{~g} / \mathrm{cm}^{2}$ to $\sim 20 \mathrm{~g} / \mathrm{cm}^{2}$ for the values of the $\mathrm{X}_{\max }$ shower maximum in the atmosphere) [11]. Upcoming LHC data studies will also help to solve the current disagreement between the observed and predicted number of muons at ground for the highest-energy showers.

In the DM sector, the LHC experiments have carried out many $\mathrm{p} p \rightarrow \chi \bar{\chi}+\mathrm{X}_{\mathrm{SM}}$ searches, complementary to those from direct measurements in underground experiments $(\chi \mathrm{N} \rightarrow \chi \mathrm{N})$ and indirect ones in space detectors $\left(\chi \bar{\chi} \rightarrow \mathrm{X}_{\mathrm{SM}} \overline{\mathrm{X}}_{\mathrm{SM}}\right)$. Searches of (R-parity conserving) SUSY at the LHC rely all in excess MET from leading DM candidates, such as neutralinos or gravitinos, for which no evidence has been found so far. The LHC has provided also the first constraints on direct Higgs boson couplings to the dark sector via $\mathrm{HZ} \rightarrow \chi \bar{\chi} l l$. The most generic DM searches in unbalanced monojet and monophoton final-states - where the DM particle is produced accompanied of initial-state QCD or QED radiation- have resulted in the lowest current limits for the $\chi-\mathrm{N}$ interaction cross-section, $\sigma_{\chi-\mathrm{N}} \approx 10^{-39} \mathrm{~cm}^{2}$ (spin independent) and $\sigma_{\chi-\mathrm{N}} \approx 10^{-41} \mathrm{~cm}^{2}$ (spin-dependent), at low dark-matter masses $\mathrm{m}_{\chi} \lesssim 10 \mathrm{GeV}$. The upcoming LHC operation in 2015, with p-p collisions at twice the energy of the first run, will provide a substantial increase in the sensitivity for dark matter searches.

\section{Acknowledgments:}

I would like to thank the organizers of ICNFP 2013 for their kind invitation to such interesting interdisciplinary meeting. 
ICNFP 2013

\section{References}

[1] D. Bauer et al., arXiv:1305.1605 [hep-ph]

[2] R. Engel, D. Heck and T. Pierog, Ann. Rev. Nucl. Part. Sci. 61 (2011) 467

[3] K. Greisen, Phys. Rev. Lett. 16 (1966) 748; G. T. Zatsepin and V. A. Kuzmin, J. Exp. Theor. Phys. Lett. 4 (1966) 78

[4] A. M. Hillas, Ann. Rev. Astron. Astrophys. 22 (1984) 425

[5] K. Werner, F. -M. Liu and T. Pierog, Phys. Rev. C 74 (2006) 044902

[6] T. Pierog, I. .Karpenko, J. M. Katzy, E. Yatsenko and K. Werner, arXiv:1306.0121 [hep-ph]

[7] N. N. Kalmykov, S. S. Ostapchenko and A. I. Pavlov, Nucl. Phys. Proc. Suppl. 52B (1997) 17

[8] S. Ostapchenko, Phys. Rev. D 83 (2011) 014018

[9] E. -J. Ahn, R. Engel, T. K. Gaisser, P. Lipari and T. Stanev, Phys. Rev. D 80 (2009) 094003

[10] D. d'Enterria et al., Astropart. Phys. 35 (2011) 98; Few Body Syst. 53 (2012) 173

[11] T. Pierog, EPJ Web Conf. 53 (2013) 01004

[12] M. Giordano, E. Meggiolaro and N. Moretti, JHEP 1209 (2012) 031

[13] G. Antchev et al. [TOTEM Collab.], Europhys. Lett. 101 (2013) 21004; Phys. Rev. Lett. 111 (2013) 012001

[14] G. Aad et al. [ATLAS Collab.], Nature Commun. 2 (2011) 463

[15] S. Chatrchyan et al. [CMS Collab.], Phys. Lett. B 722 (2013) 5

[16] B. Abelev et al. [ALICE Collab.], Eur. Phys. J. C 73 (2013) 2456

[17] S. Chatrchyan et al. [CMS Collab.], JHEP 1111 (2011) 148 [Erratum-ibid. 1202 (2012) 055]; JHEP 1304 (2013) 072

[18] R. Aaij et al. [LHCb Collab.], Eur. Phys. J. C 73 (2013) 2421

[19] G. Antchev et al. [TOTEM Collab.], Europhys. Lett. 98 (2012) 31002

[20] O. Adriani et al. [LHCf Collab.], Phys. Lett. B 703 (2011) 128

[21] P. Abreu et al. [Pierre Auger Collab.], Phys. Rev. Lett. 109 (2012) 062002

[22] M.Markevitch et al., Astrophys. J. 606 (2004) 819; D.Clowe et al., Astrophys. J. 648 (2006) 109

[23] R. P. van der Marel et al., Astrophys. J. 753 (2012) 8

[24] E. Hawkins et al., Mon. Not. Roy. Astron. Soc. 346 (2003) 78; E. Hawkins et al., Mon. Not.

Roy. Astron. Soc. 346 (2003) 78

[25] E. Komatsu et al. [WMAP Collab.], Astrophys. J. Suppl. 192 (2011) 18

[26] P. A. R. Ade et al. [Planck Collab.], arXiv:1303.5076 [astro-ph.CO]

[27] H. Baer and X. Tata, arXiv:0805.1905 [hep-ph].

[28] O. Adriani et al. [PAMELA Collab.], Nature 458 (2009) 607

[29] M. Aguilar et al. [AMS Collab.], Phys. Rev. Lett. 110 (2013) 14, 141102

[30] C. Weniger, JCAP 1208 (2012) 007

[31] S. Chatrchyan et al. [CMS Collab.], Phys. Rev. D 88 (2013) 052017

[32] G. Aad et al. [ATLAS Collab.], Phys. Rev. D 86 (2012) 092002

[33] G. Aad et al. [ATLAS Collab.], ATLAS-CONF-2013-011

[34] S. Chatrchyan et al. [CMS Collab.], CMS-PAS-HIG-13-018

[35] S. Chatrchyan et al. [CMS Collab.], JHEP 1209 (2012) 094

[36] G. Aad et al. [ATLAS Collab.], JHEP 1304 (2013) 075

[37] G. Aad et al. [ATLAS Collab.], Phys. Rev. Lett. 110 (2013) 011802

[38] S. Chatrchyan et al. [CMS Collab.], Phys. Rev. Lett. 108 (2012) 261803 